\title{
Sugars for success?
}

\author{
Ian Macdonald, MD, DSc, PhD
}

Emeritus Professor of Applied Physiology, Guy's Hospital, University of London

As long ago as the 1930s it was known that dietary carbohydrates enhance physical performance, especially in prolonged exercise. It was also known that in intense exercise over short periods the fuel used by active muscle is solely glycogen. Unfortunately, the amount of glycogen stored in muscle is limited $(800 \mathrm{kCal})$. As this glycogen is being used up, body fat has to be broken down in order to provide for the continuing need for muscle contraction. When fat becomes the prime source of muscle energy, a marked drop in muscle efficiency occurs. This not only means that more energy has to be spent to achieve the same output, but also that the individual becomes ketotic.

The aim, therefore, is to make sure the supply of muscle glycogen does not get depeleted. This applies especially to long term events. Furthermore, as the body cannot convert body fat or dietary fat to glycogen or glucose, and as dietary protein is needed for other metabolic tasks, this leaves only the carbohydrate in the diet as a major source of energy for the muscles. Carbohydrates in the diet come in many forms from complex starches to simple sugars such as sucrose, glucose and fructose.

\section{Short duration exercise}

This is usually associated with high intensity activity. The higher the intensity, the greater the muscle glycogen used and the less fat is used as an energy source; above a $\mathrm{VO}_{2}$ max of 95 per cent, glycogen exclusively is used. Thus a fast pace in the early stages of exercise will lead to rapid muscle depletion.

Moderate duration exercise of up to 60 to 90 minutes is unlikely to be influenced by carbohydrate intake during the event and so it need not be considered further. However, the presumption in making this statement is that the glycogen store in the active muscles is full of glycogen at the commencement of the moderate duration event. It should be remembered that in many short term events the athlete has to compete several times a day in heats. This will involve topping-up the muscle glycogen between events.

The value of training in short duration events, whereby fatigue is delayed and power output is improved, is due largely to the higher levels of glycogen in muscle when trained. The influence of frequent exercise, which leads to repeated bouts of muscle glycogen reduction, results in increasing the glycogen level in muscle. This glycogen must come from the carbohydrate in the diet. Furthermore, the

Correspondence: Professor I. Macdonald, Department of Physiology, Guys Hospital, University of London, London SE1 9RT, UK

C) 1990 Butterworth-Heinemann Ltd 0306-4179/90/020093-02 amount of glycogen in muscle has a direct effect on maximal power output. The success or otherwise in any event may well be decided by the levels of muscle glycogen at that time.

\section{Long duration exercise}

It has been stated that when exercise is carried out at 50 per cent $\mathrm{VO}_{2}$ max or more, complete replacement cannot occur during the exercise. But at lower levels of muscle output, the muscle glycogen can be renewed if the nutritional intake is right. Obviously, the basic source of muscle glycogen is the glucose in the blood. If the fall in blood glucose concentration which is often seen in endurance events can be prevented, then this should be encouraged. The physiological picture is complicated by insulin. The higher the level of blood glucose, the greater the output of insulin. Insulin is helpful in that it encourages muscles to take up glucose. However, it does have a disadvantage too.

A rapid output of insulin can lead to hypoglycaemia. This is obviously not conducive to good athletic performance.

\section{Carbohydrate loading}

In 1939 , it was observed that men on a high carbohydrate diet for three days performed heavy workloads for more than twice as long as men on a high fat diet for a similar period. Presumably this was due to a greater content of muscle glycogen while on the high carbohydrate diet.

Thirty years later it was proposed that the best way to achieve maximum glycogen loading in preparation for an endurance event would be first of all to deplete the muscles of glycogen by exhaustive training. The low glycogen levels would increase the activity of glycogen synthetase, an enzyme which converts blood glucose to muscle glycogen. Then, when a high carbohydrate diet was given just before the endurance competition, the muscle stores of glycogen would be higher than normal. It worked. Muscle glycogen stores were twice as high as normal and endurance was considerably increased.

The original regimen for building up these raised muscle glycogen stores was fairly drastic and gave rise to athletes becoming irritable, hypoglycaemic, unable to train and subject to overstress injuries. It has subsequently been learnt that neither the glycogen depletion by excessive exercise, nor the three days of low carbohydrate diet are necessary. Now the endurance trained athlete is able to double his muscle glycogen level by resting for two or three days while eating a diet rich in carbohydrate $(8 / 10 \mathrm{~g}$ $\mathrm{CHO} / \mathrm{kg}$ BW/day).

Glycogen in the body, whether in the liver or in muscle, is stored with about three times its weight of water. Carbohydrate loading may produce a one to two kilogram increase in body weight. It has been suggested that an 
individual's body weight, taken after emptying the bladder, may provide an indication of glycogen stores.

The limitations of this carbohydrate loading need to be spelled out. First, it is of no value for events lasting less than 90 minutes. Those lasting two to four hours are ideal for this procedure. It is unwise for older athletes, as chest pains and abnormal ECGs have been reported. It may give rise to muscle damage, severe depression and stomachache especially if the original regimen is used. There is also evidence that, if the carbohydrate loading technique is repeated too often, it fails to work. Perhaps it should be used no more than two to three times a year.

It may be that carbohydrate loading can, to some extent, be replaced by a high carbohydrate diet whose energy content is equal to that of the energy expenditure during the exercise. To eat over $500 \mathrm{~g}$ carbohydrate a day, as may be required by a $70 \mathrm{~kg}$ person may not be pleasant unless much of that carbohydrate load is provided as simple sugars, especially sucrose or as complex carbohydrates, e.g. bread, potatoes or rice.

\section{Carbohydrate feeding immediately before
exercise}

In a recent study, 10 well trained male cyclists were given, four hours before the exercise, a meal containing $200 \mathrm{~g}$ of carbohydrate in the form of cereal, bread, milk and fruit juice. Then, five minutes before exercising they were given a solid carbohydrate cereal bar. This regimen of a meal four hours before, followed by the solid carbohydrate five minutes before the event, resulted in significantly greater oxygen consumption. Of more immediate importance, it enhanced work output.

\section{During exercise}

The consumption of carbohydrates or any other nutrient during exercise has for long received the armchair criticism that all the blood goes to the muscles and that little goes to the gut. As a result, little, if any nutrient will be absorbed during muscle activity. This is good theoretical physiology, but in practice is incorrect. It was first shown several years ago when the world cross-country ski championships were won, for several years successively, by Sweden whose team had been advised to take sugars at a level greater than previously recommended during this long duration event.
It is now widely accepted that frequent ingestion of glucose, or preferably a glucose polymer, during an event is a necessity. What is open to discussion is the amount and frequency, bearing in mind that adequate water ingestion is the top priority in any endurance event and far more likely to impair performance than any lack of carbohydrate. Therefore, during exercise, unlike pre-exercise, the carbohydrate should be in solution or accompanied by water.

It has been suggested that glucose is of limited value as it seems to remain in compartments within an unavailable pool in the body during exercise. This discrepancy in opinon may be due to the strength of the glucose solution given. It seems, from several studies, that one gram of glucose polymer, not glucose as such, in $5 \mathrm{ml}$ water is physiologically and metabolically acceptable. The story about reactive hypoglycaemia may be exaggerated.

There are several reports where glucose, glucose polymer, and fructose solutions have been given at 30 minute intervals during endurance exercise. All report an improvement in performance compared with placebo, using solutions varying from 15 to 30 per cent carbohydrate. There is no doubt that glucose polymer given orally is completely available for use by active muscle during moderate to long-duration exercise.

In summary, during events lasting longer than between 60 to 90 minutes, $50 \mathrm{~g}$ of glucose polymer as a 20 per cent solution should be taken every 30 minutes by an adult. Glucose as such is not to be recommended because of its rather unpleasant taste and high osmotic pressure.

\section{Post-event carbohydrate ingestion}

Many athletes have to compete again within 24 hours or so of an event, perhaps even 12 hours later. It is essential that the muscle glycogen stores are restored to full capacity as soon as possible. The muscle glycogen store is more receptive to oral carbohydrate immediately after the end of exercise than, say, four hours later. As athletes are not keen to ingest anything immediately after an event, persuasion is required!

Thus, it is necessary to consider the following time sequences when offering advice on dietary energy intake:

Several days before the event (carbohydrate loading)

Immediately prior to the event

During the event

Immediately after the event. 